\title{
THE COMPUTATIONAL APPROACH TO COMMUTING ORDINARY DIFFERENTIAL OPERATORS OF ORDERS SIX AND NINE
}

\author{
GEOFF A. LATHAM ${ }^{1}$
}

(Received 7 August 1991; revised 12 October 1992)

\begin{abstract}
Entirely elementary methods are employed to determine explicit formulae for the coefficients of commuting ordinary differential operators of orders six and nine which correspond to an elliptic curve. These formulae come from solving the nonlinear ordinary differential equations which are equivalent to the commutativity condition. Most solutions turn out to be rational expressions in one or two arbitrary functions and their derivatives. The corresponding Burchnall-Chaundy curves are computed.
\end{abstract}

\section{Introduction}

The theory of commuting ordinary differential operators had its beginnings with the pioneering work of Burchnall and Chaundy [2, 3, 4] and Baker [1], and after some time in dormancy, has recently enjoyed a revival of interest with the application of commuting operators to completely integrable equations $[9,10,11,13,14,15,17,18,22]$. In their application to finding solutions of the Kadomtsev-Petviashvili (KP) equation for example, the basic idea is to search for solutions which lie in a special stationary manifold given by the condition of commutativity of two differential operators. It was in trying to make this idea exact that Krichever and Novikov and others were led to investigate the problem of finding explicit formulae for the coefficients of commuting operators whose orders were not coprime. The KP equation itself,

$$
\frac{3}{4} U_{y y}=\left(U_{t}-\frac{3}{4} U U_{x}-\frac{1}{4} U_{x x x}\right)_{x},
$$

'Centre for Mathematics and its Applications, ANU, GPO Box 4, Canberra ACT 2601.

(C) Australian Mathematical Society, 1994, Serial-fee code 0334-2700/94 
for a function $U(x, y, t)$ where $x$ and $y$ are space coordinates and $t$ is time, is of independent interest as a model for weakly dispersive waves in two space dimensions. The search for solutions of this equation is also the source of our motivation.

Burchnall and Chaundy showed that any two commuting ordinary differential operators $M$ and $N$ satisfy identically an algebraic relation $F(M, N)=0$ ( $F$ algebraic). We call such an $(M, N)$ a commuting pair and say loosely that the pair lies on, or corresponds to, the curve $F$. We do not review the theory of commuting operators as this is adequately done elsewhere $[2,3,4$, $12,18,19,22]$, but give a brief commentary on progress in the search for their explicit formulae. As already mentioned, of most interest in applications is the determination of the coefficients of commuting pairs $(M, N)$ for which $r:=\operatorname{gcd}(\operatorname{ord} M, \operatorname{ord} N)$ is greater than one. In this case the dimension of the common eigenspace has the possibility of exceeding one, giving a rich geometry of a higher dimensional vector bundle over the Burchnall-Chaundy curve $F$. It is a result of Krichever [12] that the general solution to this problem is parametrized by $r-1$ arbitrary functions and some arbitrary constants. Some authors [19] believe the presence of these arbitrary functions to be the main cause of the difficulty of finding explicit formulae for commuting operators.

The case of $r=1$ was solved completely by Burchnall and Chaundy and will not be mentioned further. The case where $r=2$ and $F$ determines an elliptic curve has been solved in three different ways by Dehornoy [5], Krichever and Novikov [13] (with corrections by Grinevich [7]) and Grünbaum [8]. These papers give different but equivalent forms of the coefficients of the most general commuting pair of operators of orders four and six. Both Krichever and Novikov $[13,14]$ and Grünbaum [9] have successfully applied the forms of their solutions to find associated explicit solutions of the KP equation. These authors reduce the KP equation to the so-called Krichever-Novikov equation, a completely integrable nonlinear partial differential equation in one less space dimension.

In this paper, we employ the computational approach of Grünbaum [8], to study the problem of determining explicit formulae for the coefficients of commuting pairs $\left(L_{6}, L_{9}\right)$, differential operators of orders six and nine, which lie on an elliptic curve. This might be thought of as the next most interesting case, $r=3$. In fact this problem has already been solved using geometric methods by Mokhov [16, 17] and previously Dehornoy [5] had given a partial solution. Even earlier, Dixmier [6] had given the first example of an $r=3$ commuting pair with polynomial coefficients. With the problem already solved, one might justifiably ask: why solve it again? The answer comes from the 
desire to use the explicit solution in applications, and in particular to apply it to the KP equation. From this point of view, it is desirable to give a more elementary characterization of the solution, in particular, one which avoids the appearence of elliptic functions. We offer here such an elementary approach. As justification for this attitude, we cite the success of Grünbaum in [9] for $r=2$ and the partial answer in [15] for $r=3$.

There are three main steps constituting the computational approach, which can be summarized as follows;

(i) by imposing that $F$ be elliptic, identify, and then compute, $L_{9}$ as the differential operator part of an appropriate fractional power of $L_{6}$,

(ii) compute the commutator $\left[L_{9}, L_{6}\right]$ and form the commutativity equations, which are the nonlinear ordinary differential equations in the coefficients of $L_{6}$, obtained by equating the coefficients of each power of $d / d x$ in the commutator to zero, and

(iii) solve the commutativity equations derived in (ii).

The easy statement of our problem as the simple condition $\left[L_{9}, L_{6}\right]=0$ is maligned by the rather complicated nature of the commutativity equations arising from step (ii). Although we do obtain explicit formulae for the coefficients of commuting $L_{6}$ and $L_{9}$ which depend on two arbitrary functions, we pay the price of being unable, in a computational way at least, to show that they are the most general possible. This, in part, rests on our inability to completely perform step (iii). Despite falling short on this score, the computational approach has none the less a number of advantages. First, the appearance of the arbitrary functions and constants in our solutions is very explicit. This makes the design of commuting pairs with coefficients of a particular type, for example, polynomial, rational, periodic, very easy. One has only to choose the arbitrary functions and constants appropriately. Second, Grünbaum's computational approach is simplicity itself. Nothing more complicated than the pseudo-differential calculus of I. Schur [20], based on the Leibnitz rule, is needed to carry out steps (i) and (ii). Because of this, this approach readily lends itself to implementation by computer. Finally, the method is easy. Once the commutativity equations are computed, solutions of them are relatively easy to find. This is particularly true in certain degenerate cases where all that is required is to solve second order linear differential equations.

In Section 2, we perform steps (i) and (ii) and set up the five nonlinear ordinary differential equations for the coefficients of $L_{6}$ which are equivalent to the requirement that $\left[L_{9}, L_{6}\right]=0$. It is shown that these differential equations are easily integrated once, and that all but one of five integration constants 
appearing in this process must be taken to be zero if the Burchnall-Chaundy curve $F$ is elliptic. Sections 3 and 4 are devoted to step (iii). Section 3 solves completely the case where $L_{6}$ has no second derivative term. This is one of the easy cases mentioned above. In Section 4 , the commutativity equations are further manipulated by a transformation of the last coefficient of $L_{6}$. It is then possible to obtain solutions of the commutativity equations for the case where $L_{6}$ has no first derivative term. A solution which depends on two arbitrary functions is given by reducing the five nonlinear commutativity equations to a single nonlinear integro-differential equation for one coefficient. This integrodifferential equation has appearing in its coefficients, the two arbitrary functions parametrizing the solution. Also in this section, solutions for two special cases together with some examples are given. Finally, the last section offers some comments as to the appropriateness of a major assumption used in step (iii).

\section{Commutativity equations}

Let $L_{6}$ be a general sixth order ordinary differential operator with locally smooth coefficients $a_{i}(x)$. We write this operator with the standard normalization in which the leading coefficient is 1 and the second coefficient is 0 :

$$
L_{6}=\left(D^{3}+a_{4} D+D a_{4}+a_{3}\right)^{2}+a_{2} D^{2}+D^{2} a_{2}+a_{1} D+D a_{1}+a_{0},
$$

where $D=d / d x$. We will need to work with a sixth root $L_{6}^{1 / 6}$, of $L_{6}$, in the ring of formal pseudo-differential expressions of the form $C=\sum_{-\infty}^{N} c_{i} D^{i}$. Given such an expression, define the differential operator part $C_{+}$as the sum of those terms of $C$ with nonnegative powers of $D$, and the formal integral operator part $C_{-}$, by $C_{-}=C-C_{+}$. According to the results of Schur [20], any differential operator $L_{9}$, of order 9 , which commutes with $L_{6}$ must be a constant linear combination of the operators $\left(L_{6}^{j / 6}\right)_{+}$where $0 \leq j \leq 9$. As mentioned in the introduction, any two commuting differential operators lie on some Burchnall-Chaundy curve. In this paper, we will consider only the case where the Burchnall-Chaundy curve is an elliptic curve of the form,

$$
L_{9}^{2}=L_{6}^{3}+g_{1} L_{6}+g_{0},
$$

for some constants $g_{0}$ and $g_{1}$. One can show by a careful consideration of the powers of $D$ which occur, that insisting on the curve (2.2) implies that only $j=9$ appears in $L_{9}$. We therefore take from now on:

$$
L_{9}=\left(L_{6}^{3 / 2}\right)_{+} \text {. }
$$


With this $L_{9}$, another way of writing the curve (2.2) is as

$$
L_{9}^{2}-L_{6}^{3}=-\left\{L_{9}\left(L_{6}^{3 / 2}\right)_{-}+\left(L_{6}^{3 / 2}\right)_{-} L_{9}\right\}_{+} \text {, }
$$

which is often a more convenient form for the purposes of computation. Calculating the commutator we get,

$$
\left[L_{9}, L_{6}\right]=\alpha(x) D^{4}+\beta(x) D^{3}+\gamma(x) D^{2}+\delta(x) D+\epsilon(x),
$$

where $\alpha(x), \beta(x), \gamma(x), \delta(x)$ and $\epsilon(x)$ are all differential polynomials in the coefficients $a_{i}(x)$ of $L_{6}$. If we assign the $k$-th derivative of $a_{i}, a_{t}^{(k)}$, a weight of $6-i+k$, then as is well known [22], the coefficient of $D^{j}$ in (2.5) is homogeneous of weight $13-j$. All together, these differential polynomials contain 858 terms and so it is not possible to give their expressions here. From (2.5) the condition for commutativity is, now,

$$
\alpha(x)=\beta(x)=\gamma(x)=\delta(x)=\epsilon(x)=0 .
$$

From the point of view of solving these nonlinear ordinary differential equations, it is much more useful to work not with (2.6) but linear combinations of them and their integrals and derivatives. It turns out that both $\alpha$ and $\beta-2 \alpha^{\prime}$ are exact derivatives of differential polynomials which we denote by $I_{\alpha}$ and $I_{\beta_{1}}$ respectively. Define the new differential polynomials,

$$
\begin{aligned}
\beta_{1}= & \beta-2 \alpha^{\prime}, \\
\gamma_{1}= & \gamma-3 \beta_{1}^{\prime} / 2-11 \alpha^{\prime \prime} / 6-2 a_{4} \alpha / 3+4 a_{4}^{\prime} I_{\alpha} / 3 \\
\delta_{1}= & \delta-\gamma_{1}^{\prime}-5 \beta_{1}^{\prime \prime} / 6-2 a_{4} \beta_{1} / 3+2 a_{4}^{\prime} I_{\beta_{1}} / 3 \\
& -5 \alpha^{\prime \prime \prime} / 6-2 a_{4} \alpha^{\prime} / 3+2 a_{4}^{\prime} \alpha / 3+2 / 3\left(a_{3}^{\prime}+2 a_{4}^{\prime \prime}\right) I_{\alpha}, \\
\epsilon_{1}= & \epsilon-\delta_{1}^{\prime} / 2-\gamma_{1}^{\prime \prime} / 3-2 a_{4} \gamma_{1} / 3-\beta_{1}^{\prime \prime \prime} / 6-a_{4}^{\prime} \beta_{1}^{\prime} / 3+1 / 3\left(a_{3}^{\prime}+a_{4}^{\prime \prime}\right) I_{\beta_{1}} \\
& -\alpha^{(\mathrm{iv})} / 6-a_{4} \alpha^{\prime \prime} / 3+\left(a_{4}^{\prime \prime}+a_{3}^{\prime}-a_{2} / 2\right) \alpha / 3+\left(a_{4}^{\prime \prime \prime}+a_{3}^{\prime \prime}+a_{2}^{\prime} / 2\right) I_{\alpha} / 3 .
\end{aligned}
$$

Here the prime denotes differentiation with respect to $x$. These combinations have been chosen to simplify as much as possible the expessions for $\beta_{1}, \gamma_{1}$, $\delta_{1}$ and $\epsilon_{1}$. They also preserve the homogeneity and weight, and produce exact derivatives, that is, the differential polynomials $\alpha, \beta_{1}, \gamma_{1}, \delta_{1}, \epsilon_{1}$ are the first derivatives of certain other differential polynomials denoted by $I_{\alpha}, I_{\beta_{1}}, I_{\gamma_{1}}$, $I_{\delta_{1}}, I_{\epsilon_{1}}$ respectively. In terms of these, the commutativity equations (2.6) are 
equivalent to

$$
\begin{aligned}
I_{\alpha}+K_{1} & =0, \\
I_{\beta_{1}}+K_{2} & =0, \\
I_{\gamma_{1}}+4 a_{4} K_{1} / 3+K_{3} & =0, \\
I_{\delta_{1}}+2 a_{4} K_{2} / 3+2 a_{3} K_{1} / 3+K_{4} & =0 \\
I_{\epsilon_{1}}-a_{4}^{\prime \prime} K_{1} / 9-4 a_{4}^{2} K_{1} / 9+a_{2} K_{1} / 6+a_{3} K_{2} / 3+K_{5} & =0,
\end{aligned}
$$

where the $K_{j}$ are arbitrary integration constants. We will be concerned here only with a very special choice of these constants which is forced by our assumption that, when they commute, $L_{6}$ and $L_{9}$ lie on an elliptic curve.

LEMMA 1. Assume that $\left[L_{9}, L_{6}\right]=0$ where $L_{9}$ is given by (2.3). Then $L_{6}$ and $L_{9}$ lie on the curve (2.2) if and only if

$$
K_{1}=K_{2}=K_{4}=K_{5}=0 \quad \text { and } \quad K_{3}=3 g_{1} \text {. }
$$

This is proved by explicitly expressing the coefficients of $\left(L_{6}^{3 / 2}\right)_{-}$, given that (2.8) holds, in terms of the $K_{j}$, and then forcing the form (2.2) on (2.4). We omit these somewhat complicated computations.

The constants $K_{j}$ have an interesting interpretation if we introduce a formal integral operator $\Psi=1+\sum_{1}^{\infty} \psi_{j}(x) D^{-j}$ such that $\Psi^{-1} L_{6} \Psi=D^{6}$. If coefficients $f_{j}(x)$ are defined by $\Psi^{-1} L_{9} \Psi=D^{9}-\sum_{1}^{\infty} f_{j}(x) D^{-j}$, then by conjugating the commutator $\left[L_{9}, L_{6}\right]$ with $\Psi$, it is easy to show that $L_{6}$ and $L_{9}$ commute if and only if all the $f_{j}$ are constant. In this case the commutativity equations (2.8) are satisfied, and it can be shown that $f_{j}=-K_{j} / 6$ for $j=1, \ldots, 5$. All the other $f_{j}$ are determined recursively in terms of the first five. The choice of constants given in Lemma 1 then implies that only powers of $D^{-3}$ occur in $\Psi^{-1} L_{9} \Psi$.

From now on, we adhere to the choice of constants in Lemma 1 and so will be interested in obtaining all solutions of the nonlinear differential equations represented by:

$$
I_{\alpha}=I_{\beta_{1}}=0, \quad I_{\gamma_{1}}+K_{3}=0, \quad I_{\delta_{1}}=I_{\epsilon_{1}}=0 .
$$

These equations are considerably shorter than those given by (2.6) but are still too large to put into print. To finish this section, we give the explicit expression 
for $L_{9}$ as computed from (2.3),

$$
\begin{aligned}
L_{9}= & \left(D^{3}+a_{4} D+D a_{4}+a_{3}\right)^{3}+3 a_{2} D^{5}+3\left(a_{1}+5 a_{2}^{\prime} / 2\right) D^{4} \\
& +3\left(2 a_{2} a_{4}+17 a_{2}^{\prime \prime} / 4+2 a_{1}^{\prime}+a_{0} / 2\right) D^{3} \\
& +3\left(3 a_{2} a_{4}^{\prime}+3 a_{2}^{\prime} a_{4}+2 a_{1} a_{4}+a_{2} a_{3}+31 a_{2}^{\prime \prime \prime} / 8+3 a_{1}^{\prime \prime}+3 a_{0}^{\prime} / 4\right) D^{2} \\
& +3\left(a_{2} a_{4}^{\prime \prime}-a_{2}^{\prime} a_{4}^{\prime} / 2+3 a_{2}^{\prime \prime} a_{4} / 2+2\left(a_{1} a_{4}\right)^{\prime}+a_{0} a_{4}+\left(a_{2} a_{3}\right)^{\prime}\right. \\
& \left.+a_{1} a_{3}+25 a_{2}^{(\mathrm{iv})} / 16+a_{2}^{2} / 2+2 a_{1}^{\prime \prime \prime}+9 a_{0}^{\prime \prime} / 8\right) D \\
& +3\left(-5 a_{2}^{\prime} a_{4}^{\prime \prime} / 4-a_{2}^{\prime \prime} a_{4}^{\prime}+a_{2}^{\prime \prime \prime} a_{4} / 4-a_{1}^{\prime} a_{4}^{\prime}+\left(a_{0} a_{4}\right)^{\prime} / 2-a_{2}^{\prime} a_{3}^{\prime}+\left(a_{1} a_{3}\right)^{\prime} / 2\right. \\
& \left.-a_{2}^{\prime \prime} a_{3} / 4+a_{0} a_{3} / 2+7 a_{2}^{(5)} / 32+a_{2} a_{2}^{\prime} / 2+a_{1} a_{2}+7 a_{0}^{\prime \prime \prime} / 16\right) .
\end{aligned}
$$

Again giving $a_{i}^{(k)}$ a weight of $6-i+k$, the coefficient of $D^{j}$ in $L_{9}$ is a homogeneous differential polynomial of weight $9-j$.

\section{The case $a_{2} \equiv 0$}

When $a_{2}$ is identically zero in (2.1), the commutativity equations (2.9) assume a particularly simple form. In this case, the problem of determining all commuting operators of orders six and nine reduces to the solution of second order linear differential equations, and we are therefore able to obtain the general solution. In this solution, $a_{1}$ is essentially the arbitrary function parametrizing the commuting operators.

Letting $a_{2}=0$ in (2.9) gives

$$
\begin{aligned}
& I_{\alpha}=-\frac{9}{2}\left(a_{0}^{\prime} a_{4}\right)^{\prime}-\frac{9}{2}\left(a_{1} a_{3}^{\prime}+3 a_{1}^{\prime} a_{3}\right)^{\prime}+9 a_{1}^{2}-63 a_{0}^{(\mathrm{vv})} / 16=0, \\
& I_{\beta_{1}}=6\left(a_{1} a_{4}^{(\mathrm{iv})}+5 a_{1}^{\prime} a_{4}^{\prime \prime \prime}+9 a_{1}^{\prime \prime} a_{4}^{\prime \prime}+8 a_{1}^{\prime \prime \prime} a_{4}^{\prime}+4 a_{1}^{(\mathrm{iv})} a_{4}\right) \\
& +12\left(a_{1} a_{4} a_{4}^{\prime \prime}+a_{1} a_{4}^{\prime 2} / 2+2\left(a_{1}^{\prime} a_{4}^{2}\right)^{\prime}\right) \\
& -\frac{9}{4}\left(2 a_{0}^{\prime} a_{3}^{\prime}+3 a_{0}^{\prime \prime} a_{3}\right)+6 a_{1}^{(6)}+9 a_{0} a_{1}=0, \\
& I_{\gamma_{1}}=\frac{3}{8}\left(4 a_{0}^{\prime} a_{4}^{\prime \prime \prime}+14 a_{0}^{\prime \prime} a_{4}^{\prime \prime}+21 a_{0}^{\prime \prime \prime} a_{4}^{\prime}+5 a_{0}^{(\mathrm{iv})} a_{4}\right) \\
& +3\left(3 a_{1} a_{3} a_{4}^{\prime \prime}+a_{1} a_{3}^{\prime} a_{4}^{\prime}+9 a_{1}^{\prime} a_{3} a_{4}^{\prime}-a_{1} a_{3}^{\prime \prime} a_{4}-2 a_{1}^{\prime} a_{3}^{\prime} a_{4}+3 a_{1}^{\prime \prime} a_{3} a_{4}\right) \\
& +3\left(a_{0}^{\prime} a_{4}\right)^{\prime} a_{4}+\frac{3}{4}\left(a_{1} a_{3}^{\text {(iv) }}+6 a_{1}^{\prime} a_{3}^{\prime \prime \prime}+12 a_{1}^{\prime \prime} a_{3}^{\prime \prime}+14 a_{1}^{\prime \prime \prime} a_{3}^{\prime}+15 a_{1}^{(\mathrm{iv})} a_{3}\right) \\
& +33 a_{0}^{(6)} / 32-9 a_{1} a_{1}^{\prime \prime}+9 a_{0}^{2} / 4=-K_{3} \text {, } \\
& I_{\delta_{1}}=-9 / 4\left(a_{0}^{\prime \prime} a_{1}-2 a_{0}^{\prime} a_{1}^{\prime}\right)=0 \text {, } \\
& I_{\epsilon_{1}}=3\left(a_{1}^{2} a_{4}^{\prime \prime}+2 a_{1} a_{1}^{\prime} a_{4}^{\prime}+2\left(a_{1} a_{1}^{\prime \prime}-a_{1}^{2}\right) a_{4}\right)+3\left(a_{1} a_{1}^{(\mathrm{iv})}-a_{1}^{\prime} a_{1}^{\prime \prime \prime}\right)+9 a_{0}^{2} / 16 \\
& =0 \text {. }
\end{aligned}
$$


The general solution of these equations is the next result.

PROPOSITION 2. Let $g(x)$ be an arbitrary nonconstant function and suppose that $a_{2} \equiv 0$. Define the coefficients of $L_{6}$ by

$$
\begin{aligned}
& a_{4}=-\frac{g^{\prime \prime \prime}}{2 g^{\prime}}+\frac{3\left(g^{\prime \prime}\right)^{2}}{8\left(g^{\prime}\right)^{2}}+\frac{K_{51} g+K_{52}-3 K_{41}^{2} g^{2} / 32}{g^{\prime}}, \\
& a_{3}=\frac{g^{2}-3 K_{41} g^{\prime} g^{\prime \prime} / 8-K_{41}\left(K_{51} g^{2} / 2+K_{52} g-K_{41}^{2} g^{3} / 32\right)+K_{11} g+K_{12}}{\left(g^{\prime}\right)^{3 / 2}}, \\
& a_{2}=0, \\
& a_{1}=\sqrt{g^{\prime}} \\
& a_{0}=K_{41} g+K_{42}
\end{aligned}
$$

where $K_{11}, K_{12}, K_{41}, K_{42}, K_{51}, K_{52}$ are constants. Then $\left[L_{9}, L_{6}\right]=0$ if and only if

$$
Q_{1}:=9 / 4\left(K_{41}^{2} K_{52}+8 K_{51}^{2} / 3+4 K_{42}-2 K_{11} K_{41}\right)=0 .
$$

Moreover, when (3.7) holds, the elliptic curve (2.2) is given by

$$
\begin{gathered}
g_{1}=-\left(K_{41} K_{51} K_{52}-2 K_{52}+K_{11} K_{51}+3 K_{42}^{2} / 4-9 K_{12} K_{41}^{2} / 16\right) \\
g_{0}=K_{41}^{2} K_{52}^{2}-K_{41} K_{42} K_{51} K_{52} / 2+K_{42} K_{52}-K_{11} K_{41} K_{52}-K_{11} K_{42} K_{51} / 2 \\
+3 K_{12} K_{41} K_{51} / 2-K_{42}^{3} / 4+9 K_{12} K_{41}^{2} K_{42} / 32-K_{12}+K_{11}^{2} / 4
\end{gathered}
$$

The formulae (3.6) under the condition (3.7) give the most general commuting pair for which $a_{2} \equiv 0$ and $a_{1} \not \equiv 0$.

PROOF. The labelling $K_{i j}$ generally refers to the $j^{\text {th }}$ integration constant arising in the solution of the $i^{t h}$ commutativity equation in (3.1)-(3.5). Solving (3.4) gives $a_{0}$ in terms of $a_{1}$. Next solve (3.5) for $a_{4}$, and lastly, solve (3.1) for $a_{3}$. This solves three of the equations producing (3.6), and evaluating the left hand side of (3.2) with the coefficients (3.6) gives, $I_{\beta_{1}}=Q_{1} a_{1}$, hence the algebraic condition (3.7). Substituting into (3.3) gives $I_{\gamma_{1}}=Q_{1} a_{0} / 2-K_{42} Q_{1} / 2-R_{1}$, where $R_{1}$ is a constant. We therefore take $K_{3}=R_{1}=3 g_{1}$ to obtain a solution to all the commutativity equations. The coefficients in (3.6) can now be used together with (3.7) in (2.4) to compute $g_{0}$.

Special cases of the solution (3.6) have appeared in the literature before, and we point out the correspondences. 
EXAMPLE. Let $u(x)$ be an arbitrary nonzero function and set $g^{\prime}(x)=u^{2}(x)$ in (3.6). Taking $K_{11}=K_{41}=K_{42}=K_{51}=K_{52}=0$ and $K_{12}=a$ then gives Mokhov's example (12) in [16], (see also [17, page 1307]). Further setting $u(x)=-1$ and $g(x)=x$ gives the famous example of Dixmier [6]. For both of these examples, the elliptic curve from (3.8) is, $L_{9}^{2}=L_{6}^{3}-a$.

If we take $a_{1}=a_{2}=0$ in the commutativity equations (2.9), then the situation is very simple. From (3.5), we conclude that if $a_{1}$ and $a_{2}$ are identically zero, then $\left[L_{9}, L_{6}\right]=0$ iff $a_{0}^{\prime}=0$. This solution is parametrized by the two arbitrary functions $a_{3}$ and $a_{4}$; however, we will consider this case trivial, since $L_{6}$ and $L_{9}$ are both constant coefficient polynomials in the operator $S=$ $D^{3}+a_{4} D+D a_{4}+a_{3}$. In fact, from (2.10), $L_{9}=S^{3}+3 / 2 a_{0} S$ and the curve (2.2) can be obtained by eliminating $S$.

\section{The case $a_{2} \not \equiv 0$}

The main cause of the complexity of the commutativity equations is that in general, $a_{2} \not \equiv 0$. Assuming this is so, (2.9) can be simplified somewhat by the introduction of two auxiliary functions which we now introduce.

Let $h(x)$ be any (fixed) nonconstant function such that

$$
a_{2}=h^{\prime}
$$

and define a new coefficient $u_{0}(x)$ by

$$
a_{0}=8 a_{2} a_{4} / 3+5 a_{2}^{\prime \prime} / 6-2 h^{2} / 3+u_{0} .
$$

The motivation behind this last definition comes from the case $a_{1} \equiv 0$ where it is possible to solve the equation $I_{\beta_{1}}=0$ explicitly and find precisely the relation (4.2) but with $u_{0}$ linear in $h$. As with (3.1), most of the terms in $I_{\alpha}$ are derivatives (see (4.8) below). We therefore denote by $J_{\alpha}$ the formal integral of $I_{\alpha}$, that is, the expression for which $D J_{\alpha}=I_{\alpha}$. In terms of this new integral, the first equation in (2.9) is equivalent to

$$
J_{\alpha}+K_{12}=0,
$$

where $K_{12}$ is an integration constant. Define two new expressions by

$$
\begin{aligned}
& I_{\gamma_{3}}=I_{\gamma_{1}}+\alpha^{\prime} / 6+2 h J_{\alpha} / 3, \\
& I_{\epsilon_{2}}=I_{\epsilon_{1}}+a_{2} I_{\alpha} / 6 .
\end{aligned}
$$


For the first of these, we find that

$$
I_{r_{3}}=3\left(\chi a_{3}^{\prime}+3 \chi^{\prime} a_{3}\right)+\sigma+2 a_{4} I_{\alpha} / 3
$$

where

$$
\chi=a_{1} a_{4}^{\prime}+2 a_{1}^{\prime} a_{4}+a_{2} a_{3}^{\prime}+3 a_{2}^{\prime} a_{3} / 2+a_{1}^{\prime \prime \prime}-a_{1} h,
$$

and $\sigma$ is a fairly complicated expression in which we use (4.2) to eliminate $a_{0}$ :

$$
\begin{aligned}
\sigma= & \frac{3}{8} u_{0}^{(\mathrm{vi})}+\frac{3}{8}\left(2 u_{0}^{\prime} a_{4}^{\prime \prime \prime}+9 u_{0}^{\prime \prime} a_{4}^{\prime \prime}+15 u_{0}^{\prime \prime \prime} a_{4}^{\prime}+10 u_{0}^{(\mathrm{iv})} a_{4}\right)+6\left(a_{4}^{2} u_{0}^{\prime \prime}+a_{4} a_{4}^{\prime} u_{0}^{\prime}\right) \\
& +6 a_{2} a_{4} u_{0}+\frac{21}{4}\left(a_{2}^{\prime \prime} u_{0}+a_{2}^{\prime} u_{0}^{\prime}-4 a_{2} u_{0}^{\prime \prime} / 7\right)+9 u_{0}^{2} / 4-3 h a_{4} u_{0}^{\prime}-3 h^{2} u_{0} \\
& -21 h u_{0}^{\prime \prime \prime} / 8-h^{4} / 3+6 h \int\left\{a_{1}^{2}+a_{2} u_{0}-7 a_{2}^{\prime 2} / 12+2 a_{2}^{2} a_{4}\right\}+7 a_{2} a_{2}^{\prime} h / 2 \\
& +a_{2}^{2} a_{4}^{\prime \prime}+5 a_{2} a_{2}^{\prime} a_{4}^{\prime} / 2+5 a_{2} a_{2}^{\prime \prime} a_{4}-5 a_{2}^{\prime 2} a_{4} / 2+4 a_{2}^{2} a_{4}^{2}-6 a_{1}^{2} a_{4} \\
& -9 a_{1} a_{2} a_{3}+\frac{1}{2}\left(a_{2} a_{2}^{(\mathrm{iv})}-a_{2}^{\prime} a_{2}^{\prime \prime}+a_{2}^{\prime 2} / 2\right)+a_{2}^{3}-6 a_{1} a_{1}^{\prime \prime}+3 a_{1}^{\prime 2} .
\end{aligned}
$$

Using (4.2) to eliminate $a_{0}$ from $I_{\alpha}, I_{\beta_{1}}, I_{\delta_{1}}$ and $I_{\epsilon_{2}}$ as well, we find that $a_{2}^{-3 / 2}$ is an integrating factor for most of the terms of the equation $I_{\delta_{1}}=0$. With the definitions (4.1), (4.2), (4.4), (4.5), (4.6), and (4.7), the commutativity equations (2.9) are equivalent to the following set of integro-differential equations:

$$
\begin{aligned}
I_{\alpha}= & \frac{3}{4}\left(2 a_{2} a_{4}^{\prime \prime \prime}+9 a_{2}^{\prime} a_{4}^{\prime \prime}+15 a_{2}^{\prime \prime} a_{4}^{\prime}+10 a_{2}^{\prime \prime \prime} a_{4}\right)^{\prime}+12\left(a_{2} a_{4} a_{4}^{\prime}+a_{2}^{\prime} a_{4}^{2}\right)^{\prime} \\
& +6\left(a_{2} h a_{4}\right)^{\prime}+6 a_{2}^{2} a_{4}-\frac{9}{2}\left(a_{1} a_{3}^{\prime}+3 a_{1}^{\prime} a_{3}\right)^{\prime}+3 a_{2}^{(6)} / 4 \\
& +21 a_{2}^{\prime \prime \prime} h / 4+15 a_{2} a_{2}^{\prime \prime}+9 a_{2}^{\prime 2} / 2-6 a_{2} h^{2}+9 a_{1}^{2}-63 u_{0}^{(\mathrm{iv})} / 16 \\
& -\frac{9}{2}\left(u_{0}^{\prime} a_{4}\right)^{\prime}+9 a_{2} u_{0}=0, \\
I_{\beta_{1}} / 3= & 2\left(\chi a_{4}^{\prime}+2 \chi^{\prime} a_{4}+\chi^{\prime \prime \prime}+\chi h\right)-\frac{3}{2}\left(a_{3}^{\prime} u_{0}^{\prime}+3 a_{3} u_{0}^{\prime \prime} / 2\right)+3 a_{1} u_{0} \\
= & 0 \\
I_{\gamma_{3}}-2 a_{4} I_{\alpha} / 3= & 3\left(\chi a_{3}^{\prime}+3 \chi^{\prime} a_{3}\right)+\sigma+2 h K_{12} / 3+K_{3}=0, \\
a_{2}^{1 / 2} \int a_{2}^{-3 / 2} I_{\delta_{1}} / 3= & \chi-\frac{3}{8} a_{2}^{1 / 2} \int a_{2}^{-3 / 2}\left(u_{0}^{\prime \prime} a_{1}-2 u_{0}^{\prime} a_{1}^{\prime}\right)-K_{41} a_{2}^{1 / 2}=0, \\
I_{\epsilon_{2}} / 3= & a_{1} \chi^{\prime}-a_{1}^{\prime} \chi+a_{4} H_{1}\left(u_{0}\right)+H_{1}^{\prime \prime}\left(u_{0}\right) / 8+\frac{3}{16} H_{2}\left(u_{0}\right)-\frac{5}{16} H_{3}\left(u_{0}\right) \\
= & 0,
\end{aligned}
$$

where the $H_{l}$ in the last equation are differential operators acting on $u_{0}$,

$$
\begin{aligned}
& H_{1}\left(u_{0}\right)=a_{2} u_{0}^{\prime \prime}-a_{2}^{\prime} u_{0}^{\prime}, \\
& H_{2}\left(u_{0}\right)=\left(u_{0}^{\prime}\right)^{2}+\frac{8}{3}\left(-a_{2} h u_{0}^{\prime}+a_{2}^{2} u_{0}\right), \\
& H_{3}\left(u_{0}\right)=a_{2}^{\prime} u_{0}^{\prime \prime \prime}-a_{2}^{\prime \prime} u_{0}^{\prime \prime}=\left(a_{2}^{\prime} H_{1}^{\prime}\left(u_{0}\right)-a_{2}^{\prime \prime} H_{1}\left(u_{0}\right)\right) / a_{2},
\end{aligned}
$$


and $K_{3}, K_{12}$ and $K_{41}$ are constants. Our point of view in trying to solve the commutativity equations (4.8) through (4.12) will be that $a_{1}$ and $a_{2}$ will play the role of the arbitrary functions which parametrize the solution. By this assumption, we mean that we will be searching for expressions for $a_{3}, a_{4}$ and $u_{0}$ in terms of $a_{1}$ and $a_{2}$ (and their derivatives and integrals).

An interesting feature of the new commutativity equations is the special form of (4.12). If $H_{1}\left(u_{0}\right)$ were nonzero and known in terms of $a_{1}$ and $a_{2}$, which is equivalent to knowing $u_{0}$, then (4.11) gives $\chi$ which can be put into (4.12) to give $a_{4}$ directly. Then the definition (4.6) can be used to solve a linear first order differential equation to give $a_{3}$. In this paper, we will only be concerned with the situation in which this procedure is not possible. We therefore make the following assumption for the remainder of the paper.

ASSUMPTION 4.14. Assume that $H_{1}\left(u_{0}\right) \equiv 0$.

Granted this assumption, the definition of $H_{1}$ implies that $u_{0}=K_{51} h+K_{52}$ for some constants $K_{51}$ and $K_{52}$. Substituting this $u_{0}$ into (4.11) and assuming $a_{1}$ is not proportional to $a_{2}^{1 / 2}$ gives

$$
\chi=-3 K_{51} a_{1} / 4+K_{41} a_{2}^{1 / 2} .
$$

Now putting this $\chi$ into (4.12), using (4.13) and cancelling a factor $a_{2}$ (which is nonzero), gives that

$$
\frac{3}{16}\left(K_{51}^{2}+8 K_{52} / 3\right) a_{2}-K_{41}\left(a_{1} / a_{2}^{1 / 2}\right)^{\prime}=0 .
$$

The case $a_{1} \equiv 0 \quad$ When $a_{1}$ is identically zero, we gain some simplification of the commutativity equations (4.8) through (4.12). If in addition, the integration constant $K_{41}$ is nonzero, it is easy, in this case, to obtain the most general commuting pair which depend on a single arbitrary function.

PROPOSITION 3. Let $h(x)$ be an arbitrary nonconstant function, suppose that $a_{1} \equiv 0$ and assume that (4.14) holds. Define the coefficients of $L_{6}$ by

$$
\begin{aligned}
& a_{4}=-\frac{h^{\prime \prime \prime}}{2 h^{\prime}}+\frac{3 h^{\prime 2}}{8 h^{\prime 2}}+\frac{K_{11}+\frac{3}{4} K_{51} h-\frac{1}{2} h^{2}}{h^{\prime}}, \\
& a_{3}=\left(K_{41} h+K_{42}\right) / a_{2}^{3 / 2}, \\
& a_{2}=h^{\prime}, \\
& a_{1}=0, \\
& a_{0}=-h^{\prime \prime \prime} / 2+h^{\prime 2} / h^{\prime}-2 h^{2}+3 K_{51} h+K_{52}+8 K_{11} / 3,
\end{aligned}
$$


where $K_{11}, K_{41}, K_{51}$ and $K_{52}$ are constants. Then $\left[L_{9}, L_{6}\right]=0$ if and only if

$$
Q_{1}=9\left(K_{52}+3 K_{51}^{2} / 8\right)=0 .
$$

If $K_{41} \neq 0$, then (4.17) under the condition (4.18) gives the most general commuting pair for which $a_{1}=0$ and (4.14) holds. When (4.18) is satisfied, the elliptic curve (2.2) is given by

$$
\begin{array}{cc}
g_{1}= & -\left(K_{41}^{2}+3 K_{52}^{2} / 4+4 K_{11} K_{52}+9 K_{11} K_{51}^{2} / 4+4 K_{11}^{2} / 3\right) \\
g_{0}= & K_{42}^{2}+3 K_{51} K_{41} K_{42} / 2-K_{52} K_{41}^{2} / 2-4 K_{11} K_{41}^{2} / 3 \\
& -K_{52}^{3} / 4+K_{11} K_{52}^{2}-4 K_{11}^{2} K_{52} / 3+16 K_{11}^{3} / 27
\end{array}
$$

PROOF. If $a_{1}=0$, then from either (4.11) or (4.16), $\chi=K_{41} a_{2}^{1 / 2}$, and solving this using (4.6) gives the expression for $a_{3}$. If $K_{41} \neq 0$, then substituting for $\chi$ and $a_{3}$ in (4.9) and integrating once, we obtain

$$
\left(a_{2} a_{4}\right)^{\prime}+a_{2}^{1 / 2}\left(a_{2}^{1 / 2}\right)^{\prime \prime \prime}+a_{2} h-\frac{3}{4}\left\{\frac{3}{2}\left(u_{0}^{\prime} h / a_{2}\right)^{\prime}+\frac{3}{2}\left(K_{42} / K_{41}\right)\left(u_{0}^{\prime} / a_{2}\right)^{\prime}-u_{0}^{\prime} / 2\right\}=0 .
$$

When $H_{1}\left(u_{0}\right)=0$ and $a_{1}=0,(4.16)$ gives the condition (4.18), and so $u_{0}=$ $K_{51} h-3 K_{51}^{2} / 8$. Putting this into the last relation produces the formula for $a_{4}$. At this point (4.9), (4.11) and (4.12) have been solved. The solution is then checked in (4.8) to reveal that $I_{\alpha}=Q_{1} a_{2}$, while if instead of (4.10) we use the equivalent equation $I_{\gamma_{1}}+K_{3}=0$, we find $I_{\gamma_{1}}=Q_{1} a_{0} / 2-\left(K_{52}+8 K_{11} / 3\right) Q_{1} / 2-R_{1}$ where $R_{1}$ is a polynomial given by $R_{1}=3 g_{1}$. Thus taking $K_{3}=R_{1}=3 g_{1}$ gives a solution of all the commutativity equations provided $Q_{1}=0$. Using (4.17) and (4.18) in the right-hand side of (2.4) gives the expression for $g_{0}$.

This proof uses in an essential way that $K_{41} \neq 0$, to obtain the formula for $a_{4}$. If $K_{41}=0$, then (4.9) actually implies (4.14). A statement about the generality of (4.17) in this case however, cannot be made, but some insight can be gained from the next case we treat where $a_{1}$ and $a_{2}$ are entirely arbitrary.

When (4.18) is satisfied, we get for $a_{0}$ (cf. (4.2));

$$
a_{0}=8 a_{2} a_{4} / 3+5 a_{2}^{\prime \prime} / 6-\frac{2}{3}\left(h-\frac{3}{4} K_{51}\right)^{2},
$$

so that $K_{51}$ amounts to no more than a translation of $h$. In view of (4.1), $K_{51}$ may thus seem like an irrelevant constant; however, we carry it along as it has a use in later examples. 
The case of $a_{1}$ and $a_{2}$ arbitrary In this part we operate under the assumption that $a_{1}$ and $a_{2}$ must be the two independent arbitrary functions parametrizing the commuting operators $L_{6}$ and $L_{9}$, and look for corresponding solutions of the commutativity equations. We therefore make a loose definition of independence of arbitrary functions. We will say that $a_{1}$ and $h$ (or $a_{1}$ and $a_{2}$ given (4.1)) are independent arbitrary functions if the requirement that (4.16) hold for all $x$ implies $K_{41}=0$.

If $a_{1}$ and $a_{2}$ are independent arbitrary functions, then (4.16) gives $K_{41}=0$ and $K_{52}=-3 K_{51}^{2} / 8$. We note here that there are two interesting cases when $K_{41}$ is not forced to be zero, namely $a_{1}=a_{2}^{1 / 2}$ and $a_{1}=a_{2}^{1 / 2} h$. These two cases will be considered in the next part. We now get from (4.11), $\chi=-3 K_{51} a_{1} / 4$. Substituting this $\chi$ and $u_{0}=K_{51} h-3 K_{51}^{2} / 8$ into (4.9) produces a copy of (4.11) (with $K_{41}=0$ ). Thus (4.9) and (4.11) are the same equation and so there are only three equations to solve. Since (4.11) is linear and first order in both $a_{4}$ and $a_{3}$, it can be solved for $a_{3}$ and thus this variable can be eliminated. This is the idea behind the next result.

PROPOSITION 4. Let $h(x)$ and $g^{\prime}(x)$ be independent arbitrary functions with $h^{\prime}$ not identically zero and assume that (4.14) holds. Define the coefficients of $L_{6}$ by

$$
\begin{aligned}
& a_{4}=-\frac{h^{\prime \prime \prime}}{2 h^{\prime}}+\frac{3 h^{\prime 2}}{8 h^{\prime 2}}-\frac{3 g^{\prime 2}}{2 h^{\prime 2}}+\frac{K_{11}+\frac{3}{4} K_{51} h-\frac{1}{2} h^{2}}{h^{\prime}}+v_{4}, \\
& a_{3}=-\frac{g^{\prime \prime \prime}}{h^{\prime}}+\frac{g^{\prime \prime} h^{\prime \prime}}{2 h^{2}}+\frac{g^{\prime}}{2 h^{\prime}}\left(\frac{h^{\prime \prime \prime}}{h^{\prime}}-\frac{h^{\prime 2}}{h^{2}}\right)+2 \frac{g^{3}}{h^{\prime 3}}+\frac{g^{\prime}\left(h^{2}-\frac{3}{2} K_{51} h-2 K_{11}\right)}{h^{2}} \\
& \quad-\frac{2 g^{\prime}}{h^{\prime}} v_{4}+K_{42}\left(h^{\prime}\right)^{-3 / 2}+\left(h^{\prime}\right)^{-3 / 2} \int g^{\prime}\left(h^{\prime}\right)^{-1 / 2}\left(h^{\prime} v_{4}\right)^{\prime}, \\
& a_{2}=h^{\prime}, \\
& a_{1}=g^{\prime}, \\
& a_{0}=8 h^{\prime} a_{4} / 3+5 h^{\prime \prime \prime} / 6-\frac{2}{3}\left(h-\frac{3}{4} K_{51}\right)^{2},
\end{aligned}
$$

where $K_{11}, K_{42}$ and $K_{51}$ are constants. Then $\left[L_{9}, L_{6}\right]=0$ if and only if $v_{4}$ solves the equation

$$
\begin{aligned}
0= & \left(a_{2}^{1 / 2}\left(a_{2} v_{4}\right)^{\prime}\right)^{\prime}+4 a_{2}^{3 / 2} v_{4}^{2}-4\left(h-\frac{3}{2} K_{51}\right) a_{2}^{1 / 2} h v_{4}+8 K_{11} a_{2}^{1 / 2} v_{4} \\
& -9 a_{1} a_{2}^{-1} \int a_{1} a_{2}^{-1 / 2}\left(a_{2} v_{4}\right)^{\prime}+4\left(h-\frac{3}{4} K_{51}\right) a_{2}^{-1 / 2} \int a_{2}^{2} v_{4}-9 a_{1} a_{2}^{-1} K_{42} \\
& +\left(3 K_{51} K_{11}+\frac{2}{3} K_{12}\right) a_{2}^{-1 / 2} h+\left(\frac{81}{256} K_{51}^{4}-\frac{1}{2} K_{51} K_{12}+4 K_{11}^{2}+K_{3}\right) a_{2}^{-1 / 2},
\end{aligned}
$$

with $a_{1}=g^{\prime}$ and $a_{2}=h^{\prime}$. 
PROOF. The definition of $a_{3}$ and $a_{4}$ in terms of $v_{4}$ can be derived by an iterative interplay between (4.8) and (4.11). We assume, as in the case $a_{1} \equiv 0$, that the $3 a_{2}^{(6)} / 4$ term in (4.8) is cancelled by the appropriate terms in $a_{4}$; hence $a_{4}=-a_{2}^{\prime \prime} /\left(2 a_{2}\right)+3 a_{2}^{\prime 2} /\left(8 a_{2}^{2}\right)+\cdots$. Substituting this into (4.11) gives the first few terms of $a_{3}$ and again reverting to (4.8), $a_{4}$ must have the $-3 a_{1}^{2} /\left(2 a_{2}^{2}\right)$ term to cancel the first term in $a_{3}$. From (4.11), $a_{3}$ hence gains a $2 a_{1}^{3} / a_{2}^{3}$. Continuing in this way leads to the first two expressions in (4.21) where $v_{4}$ represents unknown terms in $a_{4}$.

The independence of $a_{1}$ and $h$ implies from (4.16) that $K_{41}=0$. With $K_{41}=0, u_{0}=K_{51} h-3 K_{51}^{2} / 8$ and with $a_{3}$ and $a_{4}$ as given in (4.21), the equations (4.9), (4.11) and (4.12) are solved still with $v_{4}$ arbitrary, leaving only (4.8) and (4.10) to determine $v_{4}$. We concentrate on (4.10). Putting $\chi$ and $u_{0}$ into (4.10) gives a result which can be simplified by subtracting $K_{51} / 2$ times (4.3) from it. Doing this and evaluating the resulting equation with the above expressions for $a_{3}$ and $a_{4}$ gives the integro-differential equation (4.22) for $v_{4}$. If $L_{6}$ and $L_{9}$ are to commute, then apart from $v_{4}$ solving (4.22), any solution of this equation must give a solution of (4.8). Solving (4.22) implicitly for $v_{4}$ and substituting into (4.8) we get, $I_{\alpha}=0$ and in fact $J_{\alpha}=-K_{12}$ so the solution is consistent with (4.3).

The general solution of (4.22) seems difficult to find. A transformation of the dependent variable via $u_{4}=-2 a_{2} v_{4} / 3$ and the independent variable via $t=\int^{x} a_{2}^{-1 / 2}$ suggests that elliptic functions are involved, but this is difficult to make explicit. It also poses a considerable computational problem to compute the elliptic curve (2.2) given only that $v_{4}$ solves (4.22). We do not attempt this here; however, given that there would normally be two new integration constants entering in the solution of (4.22), it is reasonable to expect the curve to be nonsingular.

EXAMPLE. Certain obvious solutions of (4.22) are apparent. If $C$ is an arbitrary constant, then $v_{4}=C / a_{2}$ is a solution provided we choose $K_{12}=-9 / 2\left(K_{11}+\right.$ C) $K_{51}, K_{42}=0$ and $K_{3}=-\left(4\left(K_{11}+C\right)^{2}-K_{51} K_{12} / 2+81 K_{51}^{4} / 256\right)$. Hence if $C$ is taken to be nonzero, it amounts only to a translation of $K_{11}$. Thus we lose no generality by taking $C=0$, i.e. $v_{4}=0$, (and $K_{42}=0$ ) in (4.21). The elliptic curve for $L_{6}$ and $L_{9}$ is then given by

$$
L_{9}^{2}=\left(L_{6}+2 b\right)\left(L_{6}-b\right)^{2} \text {, }
$$

where $b=\left(9 K_{51}^{2}+32 K_{11}\right) / 48$. A translation of $h$ indeed shows that when $v_{4}=0$ the formulae (4.21) depend only on the single constant $b$. That (4.23) 
is singular in this case is somewhat disappointing; however, this example was used with some success in an application to the Kadomtsev-Petviashvili equation in [15]. The outcome of this work has a strong connection with the results of Painlevé analysis as done by Weiss [21].

Two special cases Here we consider separately each of the two special cases noted above. If $a_{1}$ is a linear combination of $a_{2}^{1 / 2}$ and $a_{2}^{1 / 2} h$ then (4.16) does not imply that $K_{41}=0$. If $a_{1}=a_{2}^{1 / 2}$, then $K_{41}$ remains arbitrary, while if $a_{1}=a_{2}^{1 / 2} h$, then $K_{41}$ is bound within an algebraic relation with $K_{51}$ and $K_{52}$.

If $a_{1}=a_{2}^{1 / 2}$, then the integral term in (4.11) vanishes under (4.14) to give $\chi=K_{41} a_{2}^{1 / 2}$. For all but one exceptional value of $K_{41}$, the most general commuting differential operators can easily be found.

PROPOSITION 5. Let $h(x)$ be an arbitrary nonconstant function and assume (4.14) holds. Define the coefficients of $L_{6}$ by

$$
\begin{aligned}
& a_{4}=-\frac{h^{\prime \prime \prime}}{2 h^{\prime}}+\frac{3 h^{\prime 2}}{8 h^{2}}+\frac{K_{11}+\frac{3}{4} K_{51} h-\frac{1}{2} h^{2}}{h^{\prime}}, \\
& a_{3}=\frac{h^{2}+\left(K_{41}-\frac{3}{4} K_{51}\right) h+K_{42}}{\left(h^{\prime}\right)^{3 / 2}} \\
& a_{2}=h^{\prime}, \\
& a_{1}=\sqrt{h^{\prime}} \\
& a_{0}=8 h^{\prime} a_{4} / 3+5 h^{\prime \prime \prime} / 6-\frac{2}{3}\left(h-\frac{3}{4} K_{51}\right)^{2},
\end{aligned}
$$

where $K_{11}, K_{41}$ and $K_{51}$ are arbitrary constants. Then $\left[L_{9}, L_{6}\right]=0$ and the elliptic curve is given by

$$
\begin{aligned}
g_{1}= & -\left(\frac{27}{256} K_{51}^{4}+\frac{3}{4} K_{51}^{2} K_{11}-\frac{3}{4} K_{51} K_{41}-3 K_{42}+K_{41}^{2}+\frac{4}{3} K_{11}^{2}-2 K_{11}\right) \\
g_{0}= & \frac{27}{2048} K_{51}^{6}+\frac{9}{64} K_{11} K_{51}^{4}-\frac{9}{64} K_{41} K_{51}^{3}+\frac{9}{16} K_{42} K_{51}^{2}+\frac{3}{16} K_{41}^{2} K_{51}^{2}+\frac{1}{2} K_{11}^{2} K_{51}^{2} \\
& +\frac{3}{4} K_{11} K_{51}^{2}+\frac{9}{64} K_{51}^{2}+\frac{3}{2} K_{41} K_{42} K_{51}-\frac{1}{2} K_{11} K_{41} K_{51}+K_{42}^{2}+2 K_{11} K_{42} \\
& -K_{42}-\frac{3}{8} K_{41} K_{51}-\frac{4}{3} K_{11} K_{41}^{2}+\frac{1}{4} K_{41}^{2}+\frac{16}{27} K_{11}^{3}+\frac{8}{3} K_{11}^{2} .
\end{aligned}
$$

If $K_{41}+3 K_{51} / 4 \neq 0$ then (4.24) give the most general commuting pair for which $a_{1}=a_{2}^{1 / 2}$ and (4.14) holds.

Proof. Putting $a_{1}=a_{2}^{1 / 2}$ in (4.11) gives $\chi=K_{41} a_{2}^{1 / 2}$. Using now the definition (4.6), we get

$$
\left(a_{2} a_{4}\right)^{\prime}+\left(a_{2}^{3 / 2} a_{3}\right)^{\prime}+a_{2}^{1 / 2}\left(a_{2}^{1 / 2}\right)^{\prime \prime \prime}-a_{2} h=K_{41} a_{2} .
$$


From (4.16), $K_{52}=-3 K_{51}^{2} / 8$ and hence the formula for $a_{0}$. Substituting for $\chi$ in (4.9), eliminating via (4.11) the terms which contain $a_{4}$, and cancelling a factor of $K_{41}+3 K_{51} / 4$ yields

$$
-\left(a_{2}^{3 / 2} a_{3}\right)^{\prime}+2 a_{2} h+\left(K_{41}-\frac{3}{4} K_{51}\right) a_{2}=0 .
$$

This gives $a_{3}$ and then the previous equation gives the formula for $a_{4}$. Equation (4.8) is automatically satisfied and so is (4.10) provided $K_{3}$ is taken to be three times the expression for $g_{1}$ given in (4.25). Again $g_{0}$ can be computed from (2.4).

If $a_{1}=a_{2}^{1 / 2} h$, then provided $K_{41}$ is nonzero, we can also obtain the most general commuting operators under the assumption (4.14).

PROPOSITION 6. Let $h(x)$ be an arbitrary nonconstant function and assume (4.14) holds. Define the coefficients of $L_{6}$ by

$$
\begin{aligned}
& a_{4}=-\frac{h^{\prime \prime \prime}}{2 h^{\prime}}+\frac{3 h^{\prime 2}}{8 h^{\prime 2}}+\frac{K_{11}+\frac{3}{4} K_{51} h-2 h^{2}}{h^{\prime}}, \\
& a_{3}=-\frac{3 h^{\prime \prime}}{2 h^{\prime 1 / 2}}+\frac{K_{42}+\left(K_{41}-2 K_{11}\right) h-\frac{3}{2} K_{51} h^{2}+3 h^{3}}{h^{\prime 3 / 2}}, \\
& a_{2}=h^{\prime}, \\
& a_{1}=h \sqrt{h^{\prime}}, \\
& a_{0}=8 h^{\prime} a_{4} / 3+5 h^{\prime \prime \prime} / 6-2 h^{2} / 3+K_{51} h+K_{52},
\end{aligned}
$$

where $K_{11}, K_{41}, K_{42}, K_{51}$ and $K_{52}$ are constants. Then $\left[L_{9}, L_{6}\right]=0$ if and only if

$$
K_{52}+\frac{3}{8} K_{51}^{2}-2 K_{41}=0 .
$$

If $K_{41} \neq 0$ then (4.26) under the condition (4.27) give the most general commuting pair for which $a_{1}=a_{2}^{1 / 2} h$ and (4.14) holds. When (4.27) is satisfied, the elliptic curve is given by

$$
\begin{aligned}
g_{1}= & -\left(\frac{3}{4} K_{52}^{2}-2 K_{11} K_{52}-\frac{9}{4} K_{42} K_{51}+K_{41}^{2}+6 K_{11} K_{41}+\frac{4}{3} K_{11}^{2}\right) \\
g_{0}= & -\frac{1}{4} K_{52}^{3}+K_{11} K_{52}^{2}-\frac{1}{2} K_{41}^{2} K_{52}-3 K_{11} K_{41} K_{52}-\frac{4}{3} K_{11}^{2} K_{52}-\frac{27}{64} K_{42} K_{51}^{3} \\
& +\frac{15}{4} K_{41} K_{42} K_{51}-\frac{3}{2} K_{11} K_{42} K_{51}+\frac{13}{4} K_{42}^{2}-\frac{4}{3} K_{11} K_{41}^{2}+4 K_{11}^{2} K_{41}+\frac{16}{27} K_{11}^{3} .
\end{aligned}
$$


Proof. When $a_{1}=a_{2}^{1 / 2} h$, (4.27) follows from (4.16) since again by (4.14), $u_{0}=K_{51} h+K_{52}$. Equation (4.11) gives $\chi=-\frac{3}{4} K_{51} a_{1}+K_{41} a_{2}^{1 / 2}$. Putting this $\chi$ into (4.10), using (4.27) and cancelling a factor of $K_{41}$, we find

$$
\left(a_{2} a_{4}\right)^{\prime}+a_{2}^{1 / 2}\left(a_{2}^{1 / 2}\right)^{\prime \prime \prime}+4 a_{2} h-\frac{3}{4} K_{51} a_{2}=0,
$$

which gives the expression in (4.26) for $a_{4}$. Setting (4.6) equal to the explicit $\chi$ in this case and using the formula for $a_{4}$, then gives $a_{3}$. The resulting solution is then found to solve (4.8) provided (4.27) holds, and again (4.10) gives the curve parameter $g_{1}=K_{3} / 3 . g_{0}$ once more comes from (2.4).

Each of the results in Propositions 3, 5 and 6 contain statements as to the generality of the solutions. This generality is determined by a condition on the integration constant $K_{41}$. An obvious question is to ask what happens in the cases when the condition guaranteeing generality fails. We conclude by considering this question and give some examples.

The three special circumstances of interest are:

(i) $a_{1} \equiv 0$ and $K_{41}=0$,

(ii) $a_{1}=a_{2}^{1 / 2}$ and $K_{41}=-3 K_{51} / 4$,

(iii) $a_{1}=a_{2}^{1 / 2} h$ and $K_{41}=0$.

All of these are in fact covered by the result of Proposition 4 . When $K_{41}=0$, which was assumed in the derivation of (4.22), (i) and (iii) are covered by inserting the special forms of $a_{1}$ into (4.21) and (4.22). Hence any solution of (4.22) in these cases leads to commuting operators via the formulae (4.21), and all commuting operators are obtained by solving (4.22). When $a_{1}=a_{2}^{1 / 2}$, we get $\chi=K_{41} a_{2}^{1 / 2}$. Now $K_{41}=-3 K_{51} / 4$ means that $\chi=-3 K_{51} a_{2}^{1 / 2} / 4=$ $-3 K_{51} a_{1} / 4$, which is exactly the $\chi$ used in the derivation of (4.22) from (4.21). Hence for (ii), all commuting pairs are given by solving (4.22) with $a_{1}$ replaced by $a_{2}^{1 / 2}$ and the coefficients of $L_{6}$ are given by the corresponding reduction of (4.21).

For the particular choice of $h=x$, it is easy to obtain interesting solutions of (4.22) in the above three special cases.

EXAMPLES. For each of the examples below, we fix $h=x$ and so $a_{2}=1$. For this choice, (4.22) reduces to

$$
\begin{aligned}
& v_{4}^{\prime \prime}+4 v_{4}^{2}-4\left(x-\frac{3}{2} K_{51}\right) x v_{4}+8 K_{11} v_{4}-9 a_{1} \int a_{1} v_{4}^{\prime}+4\left(x-\frac{3}{4} K_{51}\right) \int v_{4} \\
& -9 a_{1} K_{42}+\left(3 K_{51} K_{11}+\frac{2}{3} K_{12}\right) x+\frac{81}{256} K_{51}^{4}-\frac{1}{2} K_{12} K_{51}+4 K_{11}^{2}+K_{3}=0 .
\end{aligned}
$$


We give only the formula for $v_{4}$ which solves the corresponding case of (4.29) and the elliptic curve for $L_{6}$ and $L_{9}$. The formulae for $a_{4}, a_{3}$ and $a_{0}$ may be generated by substituting for $a_{1}, a_{2}, h$ and $v_{4}$ in (4.21). Our examples all have a similar form.

(i) When $a_{1}=0$ and $K_{41}=0$, the function

$$
v_{4}=-\frac{3}{2}\left(x-\frac{3}{4} K_{51}\right)^{-2}+\kappa\left(x-\frac{3}{4} K_{51}\right)^{2}-\frac{9}{32} K_{51}^{2}-K_{11}
$$

is a solution of (4.29) provided $\kappa$ takes one of the values 0 or $2 / 3$, and the constants satisfy

$$
K_{12}=\frac{81}{64}(2 \kappa-1)(4 \kappa-1) K_{51}^{3}, \quad K_{3}=-12+10 \kappa .
$$

For this case, the differential operators lie on the curve

$$
L_{9}^{2}=L_{6}^{3}+\left(\frac{10}{3} \kappa-4\right) L_{6}+K_{42}^{2} .
$$

(ii) When $a_{1}=a_{2}^{1 / 2}=1$ and $K_{41}=-3 K_{51} / 4$, the function

$$
v_{4}=-\frac{3}{2}\left(x-\frac{3}{4} K_{51}\right)^{-2}+\kappa\left(x-\frac{3}{4} K_{51}\right)^{2}-\frac{9}{32} K_{51}^{2}-K_{11}+\frac{9}{8}
$$

is a solution of (4.29) provided $\kappa$ again takes one of the values 0 or $2 / 3$ and the constants satisfy

$$
\begin{aligned}
K_{12} & =\frac{81}{64}(2 \kappa-1)(4 \kappa-1) K_{51}^{3}-81 K_{51} / 16 \\
K_{3} & =9 K_{42}-9 K_{11}+10 \kappa-111 / 16-81 K_{51}^{2} / 32
\end{aligned}
$$

The differential operators lie on the elliptic curve

$$
\begin{aligned}
& L_{9}^{2}=L_{6}^{3}+\left(3 K_{42}-3 K_{11}+\frac{10}{3} \kappa-\frac{37}{16}-\frac{27}{32} K_{51}^{2}\right) L_{6} \\
&+\left(K_{42}-K_{11}-\frac{9}{32} K_{51}^{2}\right)^{2}-5 \kappa / 2+165 / 64
\end{aligned}
$$

It is clear from both the curve and the formulae for $a_{4}$ and $a_{3}$ which come from (4.21), that one of $K_{11}$ or $K_{42}$ may be assumed to be zero since they appear only in the combination $K_{42}-K_{11}$.

(iii) When $a_{1}=a_{2}^{1 / 2} h=x$ and $K_{41}=0$, the function

$$
v_{4}=-\frac{3}{2}\left(x-\frac{3}{13} K_{51}\right)^{-2}+\kappa\left(x-\frac{3}{13} K_{51}\right)^{2}-\frac{9}{104} K_{51}^{2}-K_{11},
$$


is a solution of (4.29) provided $\kappa$ takes one of the values 0 or $13 / 6$ and the constants satisfy

$$
\begin{aligned}
K_{12} & =27 K_{42} / 2+81\left(8 \kappa^{2} / 2197-3 \kappa / 3338+1 / 208\right) K_{51}^{3}, \\
K_{3} & =10 \kappa-39+27 K_{42} K_{51} / 4+81\left(48 \kappa^{2} / 28561-35 \kappa / 8788+81 / 43264\right) K_{51}^{4} .
\end{aligned}
$$

The elliptic curve here is given by

$$
L_{9}^{2}=L_{6}^{3}+\frac{1}{3} K_{3} L_{6}+g_{0},
$$

where $K_{3}$ is as given, and

$$
g_{0}= \begin{cases}\frac{13}{4} K_{42}^{2}+K_{51}^{2}\left(\frac{3}{4}-291 K_{42} K_{51} / 832+32499 K_{51}^{4} / 4499456\right), & \text { if } \kappa=\frac{13}{6} \\ \frac{13}{4} K_{42}^{2}+K_{51}^{2}\left(\frac{27}{16}-243 K_{42} K_{51} / 832+19683 K_{51}^{4} / 4499456\right), & \text { if } \kappa=0\end{cases}
$$

It is obvious that these three examples cannot be obtained from (4.17), (4.24) or (4.26) respectively, by simply making the appropriate replacements for $a_{1}, a_{2}$, $h$ and $K_{41}$ in those formulae. These examples can also be generalized slightly by taking $h=K x$ where $K$ is a constant. If one then allows the constants to depend on extra variables $y$ and $t$, the expressions for $a_{4}$ can be made into quadratically growing single pole rational solutions of the KP equation [15].

\section{Final remarks}

All of the solutions given in the last section were obtained under the assumption (4.14), i.e. they all have $H_{1}\left(u_{0}\right)=0$. As remarked earlier, if $H_{1}\left(u_{0}\right) \neq 0$ then the possibility exists of solving (4.12) directly for $a_{4}$. To do this in a way which keeps $a_{1}$ and $a_{2}$ the arbitrary functions in the solution, we need to know $H_{1}\left(u_{0}\right)$, or equivalently $u_{0}$, in terms of $a_{1}$ and $a_{2}$. Since such an expression for $u_{0}$ is not obvious from the commutativity equations themselves, one alternative is to look for a $u_{0}$ which when put into (4.12) will produce an $a_{4}$ of a similar form to the one in (4.21). Then the expression for $a_{3}$ can be obtained from (4.11) and the solution checked in the remaining equations. For example, setting $H_{1}\left(u_{0}\right)=a_{2}^{3 / 2}$ allows us to solve for $u_{0}$ and hence $\chi$ from (4.11), and the expression for $a_{4}$ from (4.12), has the same first two terms as in (4.21). Unfortunately the resulting expressions for $a_{3}$ and $a_{4}$ do not solve the commutativity equations. Of course, there may be other choices of $H_{1}\left(u_{0}\right)$ for which 
this process does work. Support for this general idea comes from the similar looking nature of all the solutions above. They all have the form

$$
a_{4}=-\frac{f^{\prime \prime}}{2 f^{\prime}}+\frac{3 f^{\prime \prime 2}}{8 f^{\prime 2}}+\cdots,
$$

for some arbitrary function $f$. If solutions for which $H_{1}\left(u_{0}\right) \neq 0$ exist, it is reasonable to expect them also to have this form. Also, this similarity of the solutions might be explained by the use of Darboux transformations.

We have not been able to prove the existence or otherwise of solutions for which $H_{1}\left(u_{0}\right) \neq 0$. For solutions which do satisfy $H_{1}\left(u_{0}\right)=0$, the situation is no better. We have made next to no progress toward finding the general solution of (4.22). As hinted earlier, it may indeed not be possible to achieve one of the primary aims of the computational approach: the avoidance of elliptic functions. In this regard, it would be of great interest to see how the explicit formulae above compare with those of Mokhov which are written entirely in terms of the Weierstrass $\wp$-function. This seems a computationally intensive task which is beyond the current resources of the author.

It is fair to say that the computational approach here has been somewhat less successful than in the case $r=2$ [8].

\section{Acknowledgements}

This work was partly supported by Office of Navy Research grants N0001484-C-0159 and N00014-85-K-0180. The author would like to thank Alberto Grünbaum for suggesting this problem and for his supervision. Helpful discussions with Emma Previato and George Wilson are gratefully acknowledged. Thanks also go to Mike Reach for the use of his software package pspak used to perform many of the computations involving differential operators in this paper. Of course none of the results given here would have been possible without the use of Vaxima on the Vax 8800, now a 6240, at the Central Computing Services, University of California, Berkeley. The author is grateful to a referee whose suggestions improved the presentation.

\section{References}

[1] H. F. Baker, "Note on the foregoing paper 'Commutative ordinary differential operators', by J. L. Burchnall and T. W. Chaundy", Proc. Royal Soc. London A 118 (1928) 584-593.

[2] J. L. Burchnall and T. W. Chaundy, "Commutative ordinary differential operators", Proc. London Math. Soc. 21 (1923) 420-440. 
[3] J. L. Burchnall and T. W. Chaundy, "Commutative ordinary differential operators", Proc. Royal Soc. London A 118 (1928) 557-583.

[4] J. L. Burchnall and T. W. Chaundy, "Commutative ordinary differential operators II", Proc. Royal Soc. London A 134 (1932) 471-485.

[5] P. Dehornoy, "Opérateurs différentiels et courbes elliptiques", Compositio Math. 43 (1981) 71-99.

[6] J. Dixmier, "Sur les algébres de Weyl”, Bull. Soc. Math. France 96 (1968) 209-242.

[7] P. G. Grinevich, "Rational solutions for the equation of commutation of differential operators", Funct. Anal. Appl. 16 (1982) 15-19.

[8] F. A. Grünbaum, "Commuting pairs of linear ordinary differential operators of orders four and six", Physica 31D (1988) 424-433.

[9] F. A. Grünbaum, "The Kadomtsev-Petviashvili equation: an elementary approach to the 'rank 2' solutions of Krichever and Novikov", Phys. Lett. A 139 (1989) 146-150.

[10] I. M. Krichever, "Integration of nonlinear equations by methods of algebraic geometry", Funct. Anal. Appl. 11 (1977) 12-26.

[11] I. M. Krichever, "Methods of algebraic geometry in the theory of nonlinear equations", Russian Math. Surveys 32 (1977) 185-213.

[12] I. M. Krichever, "Commutative rings of ordinary differential operators", Funct. Anal. Appl. 12 (1978) 175-185.

[13] I. M. Krichever and S. P. Novikov, "Holomorphic fiberings and nonlinear equations: finite zone solutions of rank 2", Soviet Math. Dokl. 20 (1979) 650-654.

[14] I. M. Krichever and S. P. Novikov, "Holomorphic bundles over algebraic curves and nonlinear equations", Russian Math. Surveys 35 (1980) 53-79.

[15] G. A. Latham, "Solutions of the KP equation associated to rank three commuting differential operators over a singular elliptic curve", Physica 41D (1990) 55-66.

[16] I. O. Mokhov, "Commuting differential operators of rank 3 corresponding to an elliptic curve”, Russian Math. Surveys 39 (1984) 133-134.

[17] I. O. Mokhov, "Commutative differential operators of rank three and nonlinear equations", Izvestiya Akad. Nauk SSSR 53 (1989) 1291-1315 (Russian), Math. USSR Izvestiya 35 (1990) 629-655 (English).

[18] E. Previato and G. Wilson, "Vector bundles over curves and solutions of the KP equations", Proc. Symp. Pure Math. 49 (1989) 553-569.

[19] E. Previato and G. Wilson, "Differential operators and rank two bundles over elliptic curves", Compositio Math. 81 (1992) 107-119.

[20] I. Schur, "Über vertauschbare linear Differentialausdrüke", Sitz. Berliner Math. Ges. 4 (1905) 2-8, See also Ges. Abhandlungen I (Springer Verlag, Berlin 1973) 170-176.

[21] J. Weiss, "The Painlevé property for partial differential equations II: Bäcklund transformation, Lax pairs, and the Schwarzian derivative", J. Math. Phys. 24 (1983) 1405-1413.

[22] G. Wilson, "Algebraic curves and soliton equations", in Geometry today (eds. E. Arbarello, C. Procesi and E. Strickland), (Birkhaüser, Boston-Basel-Stuttgart, 1985) 303-329. 